... Denis Bron, Chef Flugmedizin der Schweizer Luftwaffe

\title{
«Ich arbeite im High-Performance-Bereich»
}

\section{Daniel Lüthi}

Text und Bilder

danielluethi[at]gmx.ch
Sein grosses Interesse liegt oben in der Luft - steht aber auch unten im Keller. Denn hier, im Untergeschoss des fliegerärztlichen Instituts in Dübendorf, finden wir sein berufliches Baby: die Unterdruckkammer, wo zunehmende Höhe simuliert wird. «Es ist die einzige in der Schweiz», sagt Denis Bron stolz.

\section{Die Realität erfliegen}

Drinnen, hinter der schweren Stahltüre, trägt er einen Pilotenhelm mit einem Verbindungsschlauch zur Sauerstoffflasche, wie seine Testkandidaten auch. «Ich gehe mit bis hinauf auf 9000 Meter», sagt er. So entsteht sehr bald der Eindruck, dass er einer von ihnen ist. Je mehr Höhe der Operateur draussen simu- liert, je weniger Sauerstoff er nach innen strömen lässt, umso mehr spüren diejenigen, die im Stahltank mit den Bullaugen sitzen, ein Kribbeln in den Armen und Beinen. Umso weniger verlässlich wird ihr Farb- und ihr Orientierungssinn, umso verlangsamter sind sie auch.

Dazu kommt im Flugzeug die Beschleunigung, «das bedeutet einen zusätzlichen riesigen Krampf», erklärt Bron. Auf dem Computerbildschirm zeigt er einen kleinen Film aus einer Zentrifuge, die in der Nähe von Dresden steht und für Tests auch Schweizer Piloten dient. Je höher die Gravitationskonstante G, desto grösser die Beschleunigung. Und die Begeisterung von Denis Bron. Der Zeiger neben dem Piloten

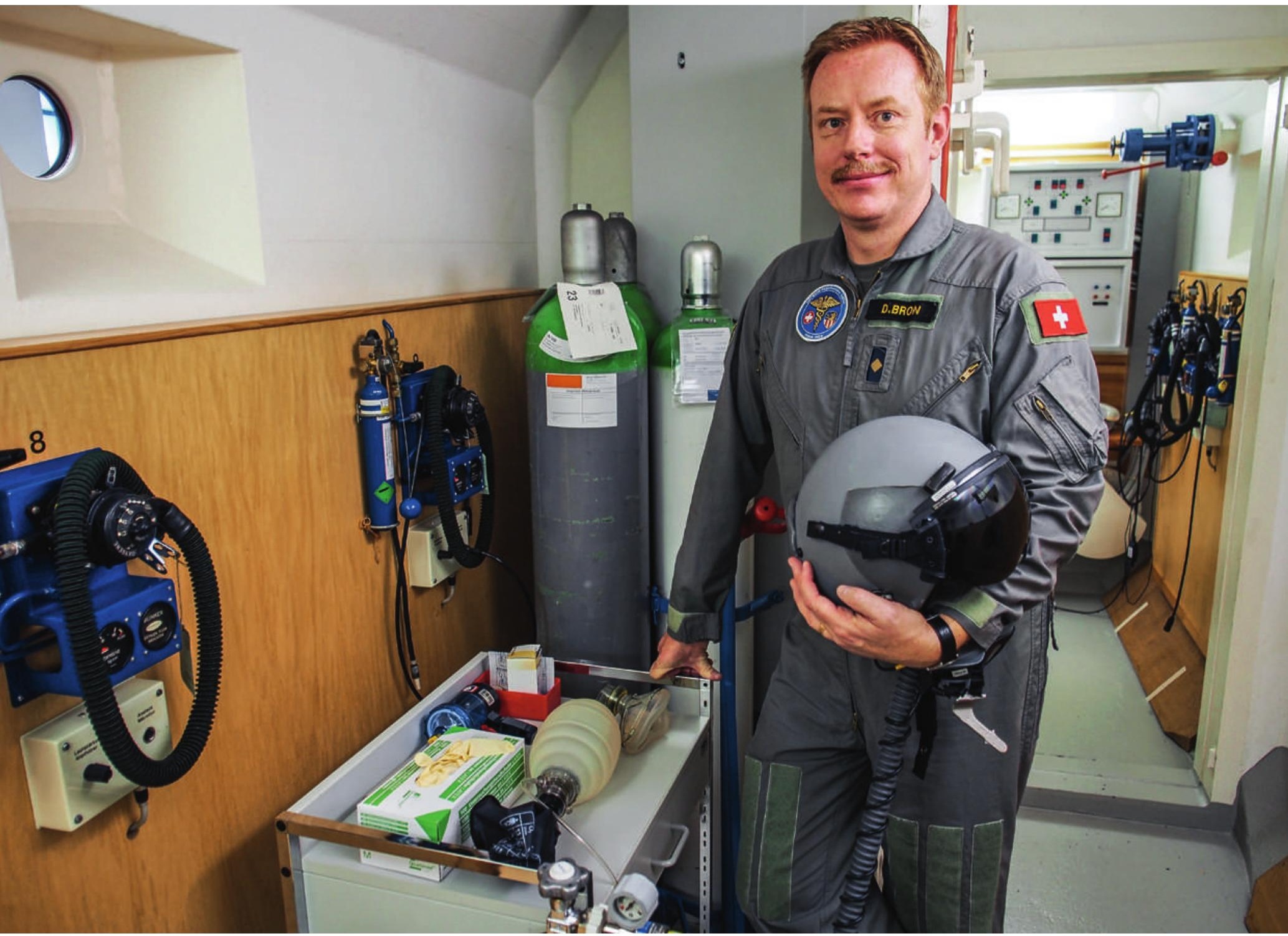


in der Zentrifuge zeigt 7 G. «Jetzt sieht man, wie's da einen zusammenstaucht», erläutert er, «unsere Leute müssen $7 \mathrm{G}$ fünfzehn Sekunden lang maintainen können. Es geht überhaupt darum, dass sie ihre Performance maintainen können, da kommt, wie im Operationssaal, während langer Zeit eine Decision nach der anderen auf sie zu.»

Immer wieder rutscht dem Aviatik-Fachmann ein englischer Fachausdruck rein. So sagt er zum Beispiel auch: «Ich muss performen, damit unsere Leute in der Luft ihre Task erfüllen können.» Und zum «performen» gehört für ihn eben auch, dass er sich der Realität seiner Klienten immer wieder selber stellt, indem er beispielsweise mit in die Unterdruckkammer geht. Und ab und zu auch in einen Militärjet. «Es ist wichtig, dass wir Mediziner manchmal mitfliegen. Ich muss selber erfahren, oder eben erfliegen, welchen Berufsrisiken unsere Piloten ausgesetzt sind. Sonst würde ich zum reinen Schreibtischtäter, der nur noch Reglemente vor sich hat.»

\section{Unmenschliches und Menschliches}

$8,4 \mathrm{G}$ habe er selber schon mal erlebt, erzählt er. Und wie immer, wenn bei ihm Stolz mitschwingt, zieht er seine linke Augenbraue nach oben. «Das war zum Glück nur ein kurzer Peak. Hätte es länger gedauert, hätte ich den Schirm wohl zugetan. Visuell wurde es schnell sehr eng, die Desorientierung war nicht zu vermeiden, mein persönlicher Bildschirm flackerte nur noch. Anschliessend war ich nudelfertig.» Eigentlich sei ein solcher Flug etwas Unmenschliches, sagt Bron, «man trägt zehn Kilogramm Material auf sich, ist in einer engen Kabine festgezurrt, kann nicht schnell aufs WC. Innerhalb von 45 Minuten verliert einer rund zwei Liter Flüssigkeit. Dazu kommen starke Vibrationen und tierischer Lärm. Und der Missionsdruck. Etwas vom Schlimmsten, was passieren kann, ist der G-Lock, also der Verlust des Bewusstseins.»

Brons Stellvertreter, ebenfalls Mediziner, war im vergangenen Oktober Passagier in einer F/A-18. Es war die Maschine, die aus bisher ungeklärten Gründen an einer Felswand des Loppers bei Alpnachstad zerschellte. «Ich war sehr betroffen», erinnert sich Denis Bron, «musste gleichzeitig aber dafür sorgen, dass der Karren weiterläuft, die Luftwaffe also weiterfliegt.» Performance eben: den Auftrag auch dann erfüllen, wenn es ganz schwierig wird. Das gilt für die Piloten ebenso, wie für ihren Arzt. Knallharte körperliche und technische Kriterien gehören bei beiden gleich dazu wie weiche Faktoren, menschliche Aspekte. Bei Denis Bron ist dies unter anderem an der Tatsache abzulesen, dass er neben einer Weiterbildung in Elektrophysiologie auch eine solche als «Human factor specialist» absolviert hat. Den Begriff «Humanfaktor» kommentiert er so: «Die Statistik besagt, dass 40 Prozent der Flugunfälle auf Pilotenfehler zurückzuführen sind, bad decision making〉. Der Unfall eines Air-France-Jumbos über dem Atlantik war typisch dafür: ein Fehlentscheid nach dem anderen - und am Schluss das Desaster.»

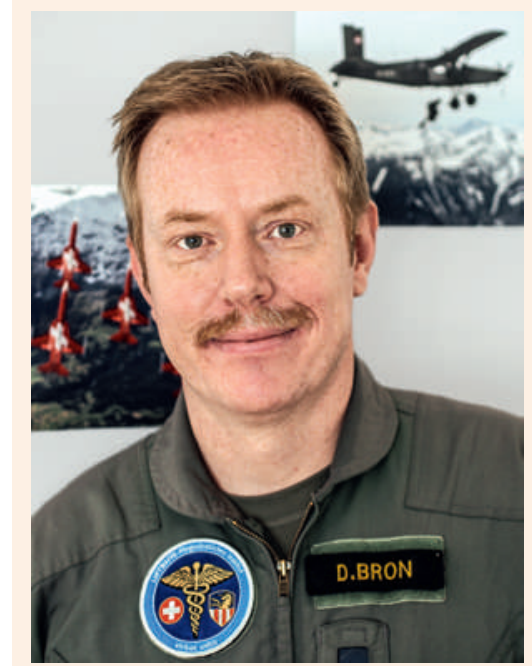

Denis Bron

Dr. med. Denis Bron wurde 1971 in Wettingen geboren und wuchs im Kanton Aargau auf, wo er auch die Schulen besuchte. Er studierte Medizin an der Universität Basel, wo er 1997 das Staatsexamen absolvierte. Neben seiner medizinischen Weiterbildung und der Arbeit an einer erfolgreichen Patentanmeldung war er im Biotech-Bereich tätig. Er publizierte unter anderem zur Muskeldystrophie Duchenne. 2004 erlangte er einen Fähigkeitsausweis in Elektrophysiologie und war unter anderem an der Harvard Universität in Boston tätig. 2013 erhielt er das Diplom als Human-Factor-Spezialist. Seit 2007 arbeitet er am fliegerärztlichen Institut der Schweizer Luftwaffe in Dübendorf. Dort ist er unter anderem zuständig für die medizinischen Aspekte bei der Rekrutierung des fliegerischen Nachwuchses sowie die medizinische Betreuung der Schweizer Militärpiloten, der Fallschirmaufklärer und der Flugverkehrsleiter. Denis Bron ist verheiratet und Vater von drei Kindern. Er lebt mit seiner Familie in Bözberg/AG.

\section{Höchstform als Pflicht}

Eine praktisch makellose physische und psychische Konstitution und Kondition sind obligatorische Voraussetzungen für den Pilotenberuf. Oder wie es in einer der Broschüren heisst, die im fliegerärztlichen Institut aufliegen: «Höchstform ist Pflicht». Der Schritt zu romantischen Übersteigerung ist nahe, Attribute wie «Superman» oder «Held der Lüfte» liegen schnell auf der Zunge. Die Leute, die in den Gängen des fliegerärztlichen Instituts anzutreffen sind, wirken jedoch bescheiden, offensichtlich fühlen sie sich wie Soldaten, die nur ihre Pflicht erfüllen. So auch Denis Bron, der von der Luftwaffe, also vom VBS angestellt ist und bisweilen auch eine Uniform mit dem Gradabzeichen eines Majors trägt, eigentlich aber ein Zivilist ist. «Ich arbeite im High-Performance-Bereich», 
sagt er. «Es gelten hier aber ähnliche Gesetze wie im Low-Performance-Bereich. Hier und dort geht es um Muskelstärke, um Kraft.» Er muss es wissen, denn lange arbeitete er am anderen Ende des Spektrums, war spezialisiert auf eine schwere körperliche Behinderung, die Erbkrankheit «Muskelschwund Duchenne». Sie schreitet rasch voran und endet immer mit dem Tod, meist schon im Jugendalter. Als sich Bron mit dieser Krankheit beschäftigte, interessierten ihn vor allem deren biochemischen Aspekte. «Als Arzt kam ich nicht bis zur Ursache, ich konzentrierte mich also auf die Therapie, wollte wissen, wie ich einen Wirkstoff schneller ans Zielorgan bringen kann. Schon während meiner Assistenzzeit machte ich zu Hause entsprechende Badewannen-Versuche.» Später leitete er in diesem Bereich dann ein Biotech-Unternehmen.

\section{Der eigene Makel}

Eine riesige Schere, die da zwischen stahlharten Supermen und sterbenden Kindern plötzlich aufgegangen ist. «Ich war immer ein Between», sagt Denis Bron dazu. So sitzt er auch regelmässig in Militärjets, ist selber aber nie Pilot geworden. «Das war gar nicht möglich, weil ich selber eine Rot-Grün-Schwäche habe, ein absolutes No-Go.» Den Traum hätte er schon gehabt. «Ich erinnere mich gut, wie ich als kleiner Knabe fasziniert war, als mir die Hunter um den Sandkasten flogen.»

Jetzt arbeitet Bron also auch bei der Luftwaffe, hat aber eine ganz andere Mission als die Berufsleute, für die er medizinisch verantwortlich ist. Rund 300 Piloten gehören dazu und 100 Fallschirmaufklärer, aber auch Flugverkehrsleiter. Und all die jungen Kandidaten, die via die Organisation SPHAIR ihren Traum zum Beruf machen und Pilot werden wollen. Das Interesse von Frauen sei leider sehr klein, sagt Bron als medizinischer Leiter dieses Auswahlprozesses, Militärpilotinnen gebe es in der Schweiz nur eine Handvoll, darunter keine einzige Jetpilotin. «Es wird immer schwieriger, guten Nachwuchs zu rekrutieren. Ich sehe eine Bürotisch-Generation. Dazu werden die Leute immer individualistischer, bei uns aber spielt das Team eine grosse Rolle.» Das Stoffemblem auf der Brust unterstreicht diese Aussage: «viribus unitis» heisst es da: mit vereinten Kräften

$\mathrm{Zu}$ den Faktoren, die das Metier hier schwierig machen, kommt die Herausforderung zu erklären, warum die neutrale, friedliche Schweiz eine Luftwaffe braucht. «Für viele Junge sind Konflikte wie derjenige in Syrien eben sehr weit weg.» Für Denis Bron sind sie reale Bedrohungen. «Ja, die Verteidigungsfähigkeit der Schweiz ist für mich eine Notwendigkeit.»

\section{Qualität als Leitlinie}

Sein normaler Alltag ist viel profaner. Dazu gehört zum Beispiel ein Pilot mit Rückenschmerzen. Oder mit einer Scheidungsproblematik, die zu viel Aufmerksamkeit verlangt. Die Grippe eines Flugverkehrsleiters und die Frage, ob er fit genug ist für seine verantwortungsvolle Aufgabe. «Wir müssen eine Vertrauensbasis schaffen, damit die Leute mit allem frühzeitig hierher kommen und wir rechtzeitig eingreifen und den maximalen Support geben können.» Und auch hier ist immer wieder ein schneller und klarer Entscheid gefragt: «Ich war auch schon dafür verantwortlich, dass ein Swiss-Flug gecancelt wurde, weil ich befand, dass der Pilot physisch und psychisch nicht auf der Höhe seiner Kräfte war.»

\section{«lch war auch schon dafür verantwortlich, dass ein Swiss-Flug gecancelt wurde.»}

High-Performance ist die Leitlinie. «Höchste Qualität ist meine Mission. Die Piloten sollen möglichst lang in der Luft bleiben, sollen ihren Beruf also möglichst lange ausüben können. Das spielt bei der Auswahl eine Rolle, dann aber auch im Berufsalltag. Die Realität des Gesundheitswesens aber sieht anders aus. Sie ist money-driven. Die Kosten stehen im Vordergrund, was bei uns nicht der Fall sein darf. Flugmedizin ist eine ganz spezielle Ecke. Der TARMED hilft uns nicht weiter. Vielleicht deshalb existieren wir Flugmediziner hierzulande in der medizinischen Landschaft faktisch gar nicht; in Deutschland und Frankreich gibt es das Fachgebiet offiziell, in der Schweiz nicht.» Ein «leider» schwingt gut spürbar mit.

Übrigens: Dass er selber nicht hat Pilot werden können, hat Denis Bron gut verdaut. Für seinen zehnjährigen Sohn, der auch vom Fliegen träumt, sind die Chancen indessen intakt. «Den Farbsinn habe ich bei ihm schon abgeklärt», sagt Vater Bron. Und zieht einmal mehr seine linke Augenbraue nach oben.

\section{Die nächste «Begegnung mit ...»}

Am Ende jeden Monats stellt die Schweizerische Ärztezeitung eine Persönlichkeit vor, die sich im Gesundheitswesen engagiert. Im Juni schildert Daniel Lüthi seine Begegnung mit Nadia Khan, Neurochirurgin und Leitende Ärztin für Moyamoya am Kinderspital Zürich. 